FUNCION DE LOS HERBARIOS EN LOS ESTUDIOS PALEONTOLOGICOS, PALEOBOTANICOS, ESTRATIGRAFICOS, PALEOCLIMATICOS Y PALEOECOLOGICOS

\author{
Jaime Rueda Gaxiola* \\ Eloy Salas*
}

\title{
LA PALEOBOTANICA
}

La Paleobotánica, como una rama de la Paleontología, nació en Francia entre los años de 1820 a 1830, fundada por Adolfo Brongniart. En un principio tuvo un carácter de ciencia eminentemente descriptiva, mas poco a poco llegó a ser una ciencia de grandes e importantes aplicaciones, siendo unas de carácter botánico orientadas al conocimiento de las plantas fósiles con el fin de resolver los problemas de evolución, por medio de la morfología comparada, para reconstruir la anatomía de las plantas desaparecidas, base de la clasificación natural de los vegetales. Otras de carácter geológico, orientadas a la determinación de edades de las rocas que contienen los fósiles, al estudio de la distribución de los vegetales en el pasado y al conocimiento del origen de algunas rocas formadas por vegetales y del clima existente en los diferentes períodos geológicos.

De acuerdo con el tamaño de los restos fósiles en estudio, la Paleontología se divide en dos partes: la Paleomacrontología y la Paleomicrontología. La primera estudia organismos, órganos y fragmentos orgánicos fósiles de más de 1 cm; la segunda de menos de $1 \mathrm{~cm}$. Dentro de los fósiles estudiados por la Paleobotánica tenemos tanto macro como microfósiles. De acuerdo con el tipo de planta de que se trate, el estudio de la planta entera, o de alguna de sus partes, caerá dentro del dominio de la Paleomacrobotánica o de la Paleomicrobotánica. Así, dentro de la segunda se estudian fragmentos orgánicos vegetales, como cutículas; órganos, como esporangios y organismos, como las algas microscópicas.

Pero ¿Cómo se han preservado estos vestigios vegetales en los sedimentos? Existen dos tipos de restos vegetales; los fósiles propiamente dichos y los subfósiles. En los primeros se ha efectuado todo un proceso de fosilización en que

* Instituto Mexicano del Petróleo. México, D. F. 
la acción de varios tipos de agentes de un determinado medio han hecho que los restos pierdan su contenido orgánico; los segundos todavía se encuentran experimentando la acción de tales agentes y tienen un gran contenido orgánico.

Debido a su constitución muy especial, los vegetales cuando mueren son destruidos con cierta facilidad por agentes de tipo mecánico, químico o biológico. Esto es particularmente cierto en vegetales de ambientes continentales que no están cubiertos por capas calcáreas o silíceas secretadas por los mismos para su protección, como es el caso de algunas plantas acuáticas microscópicas.

En los vegetales continentales se efectúa una desintegración aún cuando la planta esté viva. Partes de la planta se separan debido a diferentes cambios durante la vida del vegetal. Muchos de estos cambios son debidos al clima (pérdida de las hojas por ejemplo), otros debidos a procesos reproductivos (pérdida de flores, polen, esporas, frutos etc.). Todas estas partes sufren de inmediato, al separarse de la planta, modificaciones debidas a agentes del medio que generalmente las destruyen. Se necesitan condiciones realmente ideales para que la mayoría de los vegetales se fosilicen.

Las limitaciones anteriores han impedido en gran parte el desarrollo de la Paleobotánica, ya que no existen grandes yacimientos de macrofósiles vegetales en el mundo que permitan estudiar con facilidad las diferentes partes de ellos y hacer una reconstrucción adecuada de los mismos.

Los macrofósiles generalmente se conservan en forma de: compresiones, impresiones, moldes, petrificaciones, carbonizaciones y momificaciones. En los tres primeros tipos de fosilización, el vegetal pierde su estructura interna y no queda de él más que la forma externa y en el caso de los moldes es posible determinar también el volumen. Las petrificaciones pueden ser de diferentes tipos: carbonatación, silicificación, piritización, etc. En los casos de petrificación, la estructura del vegetal es muchas veces conservada, debido a que el proceso se efectúa muy lentamente en forma de un intercambio de moléculas de materia orgánica por materia mineral. Otros organismos vegetales han formado caparazones o conchas, secretando sustancias minerales que cubren sus partes blandas. Plantas de este tipo son algunas algas como las carófitas, las diatomeas y otras que en ocasiones son formadoras de rocas de origen orgánico. La carbonización es otro tipo de fosilización llevada a cabo por la disminución de los constituyentes volátiles de los tejidos originales $(\mathrm{O}, \mathrm{H}, \mathrm{N})$ quedando una delgada película de carbón con las estructuras del ejemplar y son frecuentemente encontradas en los depósitos de hulla.

En general, un medio adecuado para que el material vegetal se encuentre conservado, es aquel cuerpo de agua protegido de los vientos y sin fuertes 
corrientes como un pequeño lago, bahía o pantano en que se acumulen sedimentos de grano fino en forma tan rápida que produzcan un enterramiento, que haya baja cantidad de oxígeno y sustancias tóxicas que retarden la descomposición de la materia orgánica.

Por el contrario, existen microfósiles vegetales que debido a su constitución orgánica, se conservan en perfectas condiciones a través del tiempo geológico, por medio de momificaciones, dentro de diferentes tipos de sedimentos, sin cambiar radicalmente su composición original. Estos microfósiles llamados orgánicos pertenecen a restos vegetales, órganos y aún organismos compuestos por sustancias sumamente resistentes del tipo de los polímeros tales como la cutina, la quitina y la esporopolenina. Estas sustancias constituyen las cutículas de los frutos y de las hojas, forman parte de los tejidos leñosos de algunas plantas superiores como son las traqueidas o bien constituyen la membrana protectora de los granos de polen y de las esporas. Finalmente, muchos vegetales microscópicos acuáticos están protegidos por membranas formadas por sustancias quitinosas. Estos microfósiles vegetales, debido a su gran abundancia, a su gran difusión y sobre todo a su gran resistencia a los agentes destructivos de la materia orgánica, se encuentran distribuídos desde hace miles de millones de años en los sedimentos, siendo mudos testimonios de la evolución biológica. A diferencia de los macrofósiles vegetales, se les encuentra en todos los tipos de sedimentos, tanto marinos como continentales y en todas las latitudes. El estudio de estos microfósiles orgánicos, resistentes a la acción de ácidos tan activos como el fluorhídrico, el clorhídrico, el nítrico, etc. es el dominio de la parte de la Paleomicrontología Vegetal llamada Paleopalinología. Esta ciencia estudiaba al principio los microfósiles orgánicos vegetales, pero poco a poco fue ampliando su campo de acción hacia la Paleomicrontología Animal curando se encontró junto a los microfósiles orgánicos vegetales, otros microfósiles orgánicos animales.

Como hemos visto anteriormente, los fósiles vegetales son generalmente fragmentarios. El investigador de estos restos deberá siempre, o casi siempre, estudiar restos macroscópicos y/o microscópicos que forman sólo una pequeña parte de una planta completa. Unicamente cuando se trata de micro-organismos vegetales, el investigador trabaja con una entidad casi completa, pues frecuentemente conservan la parte externa.

Cuando quiere reconstruir la planta original, en base a estos fragmentos, siempre encuentra grandes dificultades, debido a que raramente se presentan en un yacimiento todas las partes de su rompecabezas. Es entonces muy importante que el investigador tenga los conocimientos generales sobre las plan- 
tas del presente y del pasado que le permitan fundar sus hipótesis científicas de trabajo. Por lo tanto, la persona que vaya a dedicarse a la paleobotánica debe primero estudiar aquellos ejemplares de herbario cuyo registro fósil se conoce y que estén representados en la actualidad, como sucede con numerosas familias del Terciario y con los llamados fósiles vivientes domo Ginkgo, Araucaria, etc., que vienen desde muy atrás en el registro geológico.

Si el presente es la clave del pasado, no es menos cierto que los fósiles pueden darnos también la clave del presente. Los procesos evolutivos están grabados en los fósiles micro y macroscópicos. Es necesario únicamente emplear principios lógicos y conocimientos científicos básicos para encontrar en estos fósiles los datos necesarios de su evolución.

Los estudios paleontológicos siempre han sido básicamente morfológicos. La Paleobotánica desde sus orígenes fue morfológica; comparando la forma de los fósiles con las plantas actuales, Brongniart determinó el origen de ellos. Esta comparación de los seres actuales con los del pasado es una de las técnicas más antiguas de la paleontología. Para obtener los mejores resultados es necesario que estas comparaciones se hagan tomando como base los mejores materiales actuales y fósiles. Cuanto mayor sea el número de comparaciones de un determinado fósil, con ejemplares actuales, mayor será el número de posibilidades de encontrar el grupo botánico al cual el fósil pertenece o se asemeja. Este método elemental de comparación es muy laborioso, pero bastante seguro si se cuenta con una colección de ejemplares lo suficientemente grande y bien clasificada.

Un herbario debería, entonces, contener no únicamente material seco y comprimido, sino contar también con grandes colecciones de plantas microscópicas. Es más, creemos que debe también contar con colecciones de macrofósiles y microfósiles vegetales y con un archivo de fotomicrografías de estos últimos.

\section{FUNCION DE LOS HERBARIOS EN LOS ESTUDIOS ESTRATIGRAFICOS}

La estratigrafía como parte de la Geología que estudia los estratos de la corteza terrestre, estudia también la composición de los mismos, así como su distribución geográfica, tratando de establecer su sucesión cronológica por medio de la determinación de edades relativas y absolutas. De acuerdo con esto vemos que la estratigrafía está basada en diferentes ramas de las ciencias naturales. Por un lado estudia las rocas principalmente sedimentarias (Litoestra- 
tigrafía) desde el punto de vista de su origen por lo que se relaciona con dos ciencias: la Sedimentología y la Petrología. Por otra parte, trata de determinar la edad de los estratos (Cronoestratigrafía) por medio principalmente de estudios de los fósiles contenidos en ellos (Bioestratigrafía) y también su distribución geográfica, por lo que está muy relacionada con otras ciencias tales como la Paleontología y la Paleogeografía.

La edad relativa de los estratos está dada por un principio sencillo, expuesto desde 1869 por el médico danés N. Steno: en una serie sedimentaria no deformada, las capas se sobreponen por edad progresiva. Sin embargo, este principio no es válido cuando estamos en presencia de series que han sido deformadas por movimientos tectónicos, desconociéndose su posición original. En estos casos es necesario, entonces, aplicar otro criterio basado en un principio, expuesto en 1800 por el inglés W. Smith, según el cual los fósiles tienen una repartición fija en las capas geológicas, permitiendo caracterizarlos por medio de su fauna y de su flora fósiles y por lo tanto determinar su sucesión. Estos fósiles, nos ayudan a comprender, según su complejidad, su distribución en el tiempo y en el espacio, la edad y los medios ambientes en que vivieron, ayudando a entender también el origen de los sedimentos y su distribución geográfica. Aunque generalmente se cree que los fósiles únicamente tienen importancia como marcadores de edades y como indicadores de ambientes, también tienen una gran importancia relacionada con la formación de las rocas (Litoestratigrafía) ya que muchas de ellas son formadas por organismos animales o vegetales, sea directa o indirectamente. Dentro de las rocas formadas por vegetales tenemos el carbón mineral y sus variedades, formado por la alteración química, física y biológica de organismos, órganos y fragmentos orgánicos vegetales macro y microscópicos; la diatomita, roca silícea formada por la acumulación de diatomeas; rocas carbonatadas formadas por la acumulación de Cocolitofóridos. Otras rocas están constituídas por algas calcáreas como Diplopores y Lithothamnium; rocas formadas por concreciones calcáreas llamadas estromatolitos originados por la actividad de algas azules del tipo de Cryptozoon, etc. Es así como se destaca la importancia de las plantas dentro de la Bioestratigrafía y de la Cronoestratigrafía. Todos los cambios evolutivos han quedado registrados en los sedimentos durante la historia de la tierra desde el Precámbrico lo que nos permite determinar la edad de los sedimentos en base al grado de evolución de los vegetales encontrados en ellos. Para esto es necesario conocer el alcance estratigráfico de cada una de las plantas fósiles y su distribución geográfica. Estos cambios evolutivos están generalmente representados morfológicamente en los macro y microfósiles vegetales. 


\section{EL USO DE LOS CARACTERES MORFOLOGICOS DE LOS FITOFOSILES EN ESTUDIOS PALEOCLIMATICOS Y PALEOCOLOGICOS}

La Paleoclimatología es el estudio del clima a través del pasado geológico por lo que está relacionada con dos factores principales: el tiempo y el espacio. El conocimiento del clima presente en diferentes latitudes es fácilmente cuantificable y de estos datos se deducen otros para el estudio de climas pasados. Existen innumerables criterios que nos permiten deducir un paleoclima en base a observaciones de tipo geológico: factores litológicos tales como la presencia de ciertos tipos de arena, arcillas, de rocas evaporíticas, de suelos, de lechos rojos, de marcas de desecación, etc. y factores biológicos tales como macro y microfósiles vegetales y animales.

Las plantas son algunas veces llamadas los termómetros del pasado. Esto es verdad cuando se conocen los límites de temperatura que soporta una planta. Si podemos asociar una planta fósil con su representante actual, podremos deducir a partir de las condiciones ambientales de la especie moderna, aquéllas que pudieron haber subsistido en el pasado. Aquí vuelve a cobrar importancia el tener una fuente de comparación que permita clasificar el ejemplar fósil utilizando sus representantes de herbario actuales. Desafortunadamente las especies vivientes decrecen en número conforme nos adentramos en el pasado y así las condiciones climáticas no pueden inferirse con tanta seguridad en rocas más antiguas que las del Terciario.

La naturaleza de los fósiles vegetales encontrados en los sedimentos y su estudio comparativo con las plantas actuales, han predeterminado, según Dorf (1970), los tres métodos utilizados en la determinación de los paleoclimas a saber:

1.-Los climas del Cuaternario están basados en estudios casi exclusivamente de microfósiles vegetales, en particular de polenesporas. * En este caso las polenesporas encontradas en los sedimentos permiten hacer una determinación y clasificación dentro de taxa modernos de los curales se conoce perfectamente el clima en donde viven actualmente. Las variaciones climáticas del Cuaternario han servido para subdividir este período geológico en épocas y en edades basadas en glaciaciones e interglaciaciones.

Desgraciadamente, los restos macroscópicos de las Angiospermas corresponden principalmente a plantas leñosas. Las hierbas están muy pobremente representadas, excepto palinológicamente.

* Este término, en francés e inglés Pollenospores, es empleado en español para indicar indistintamente tanto polen como esporas. 
El período pleistocénico tuvo una serie de características fitogeográficas interesantes: un clima muy peculiar y floras muy bien conocidas debido principalmente a su íntima relación con las plantas recientes que pueden ser comparadas fácilmente en una área dada, con ejemplares de herbario. Indirectamente los numerosos diagramas polínicos hechos en este período han permitido seguir la pista de las migraciones de muchas especies de árboles a través del tiempo.

2.-Para determinar los paleoclimas del Cretácico Medio al Terciario, se han empleado sobre todo los macrofósiles vegetales, principalmente hojas de Dicotiledóneas, ya que este grupo de plantas representa dentro de las actuales el de mayor índice de confianza. Las relaciones de estos fósiles con los taxa actuales, sin embargo, son cada vez menores conforme nos alejamos del presente. Los microfósiles vegetales cada día son más empleados para este tipo de interpretaciones climáticas ya que por lo general están mejor representados, y en mayor cantidad, que los macrofósiles.

3.-Para la determinación de los paleoclimas pre-cretácicos, se emplean macrofósiles de Coníferas, Cycadales y Ginkgoales alejados de cualquier comparación, excepto con plantas actuales de los llamados fósiles vivientes. Es lógico que si retrocedemos hasta el Paleozóico, habrá cada vez menor número de formas fósiles vegetales y también menor grado de comparación con las plantas actuales. Así, tendremos que basarnos únicamente en restos de planta: relacionadas con Pteridófitas, Briófitas, Algas, etc. que nos indicarán en el mejor de los casos únicamente ambientes con clima húmedo o acuático.

Para la aplicación del método de comparación entre el material fósil y el de un herbario, es necesario tener en mente que los resultados, conforme nos alejamos del presente, serán afectados por factores como los siguientes, ya citados por Dorf en 1970:

1.-Las condiciones climáticas actuales son aparentemente anormalmente frías, al igual que las rápidas fluctuaciones climáticas del Cuaternario debidas a glaciaciones.

2.-Las plantas pueden haber cambiado sus requerimientos climáticos durante el Terciario.

3.-Las comunidades vegetales pueden emigrar más rápidamente que adaptarse a las fluctuaciones del medio ambiente incluyendo las climáticas.

4.- - Una colección grande de plantas fósiles de una localidad dada generalmente representa la vegetación que creció en la vecindad de la cuenca de depositación de esa localidad.

Estos factores afectan los resultados en estudios de macro y microfósiles, 
aunque es justo reconocer que la Paleomicrobotánica permite comparar mayor número de ejemplares de mayor número de especis y que en general en una cuenca se encuentran depositados tanto microfósiles vegetales autóctonos como alóctonos, permitiendo saber en muchos casos el clima reinante tanto alrededor de la cuenca como en los lugares circunvecinos. Según Dorf, para inferir el clima en base a macrofósiles, es necesario colectar un mínimo de 25 especies, en cambio para análisis estadísticos de las características morfológicas de los restos vegetales, son necesarios al menos 500 ejemplares, preferentemente 1000 .

Otros factores que afectan la representación numérica de los fósiles vegetales en un yacimiento determinado son, según Chaney, 1959 (in E. Dorf, 1970):

1.-Factores de depósito tales como: Proximidad de las plantas al lugar de depósito, preservación de los órganos a través del tiempo, peso por área de los órganos fosilizados, altura y volumen de las plantas madres de estos órganos y el hábito caducifolio o perennifolio de las plantas madres.

2.-Factores que impiden la identificación de algunos órganos, lo que ocasiona que se ignoren o se clasifiquen mal.

3.-Factores de corrección para la restauración de los fósiles, pues las unidades encontradas en tına flora fósil no representan el total de los órganos de las plantas madres.

Estos factores son igualmente válidos para estudios de macro y microfósiles vegetales, pero, una vez más, la Palinología ha avanzado en cuanto al empleo de estos factores de corrección, debido a que los microfósiles vegetales son encontrados en grandes cantidades en diferentes condiciones de depósito, lo que permite emplear innumerables métodos estadísticos y de computación electrónica. Sin embargo, también la Palinología tiene sus problemas en cuanto a la determinación de paleoclimas, pues aunque la casi totalidad de los granos de polen y de las esporas recientes pueden ser identificadas a nivel genérico, muchas no pueden ser determinadas a nivel específico. Felizmente, la Palinología cuenta con grandes recursos para ir poco a poco diferenciando las especies de los diferentes géneros por medios tan sofisticados como el uso del microscópico de barrido electrónico que permite separar las especies en base al aspecto externo de las polenesporas aún cuando estas caracteristicas no puedan ser vistas al microscopio fotónico, ni al electrónico (Heim \& Perreau, 1971; Reyre, 1971; Sylvester-Bardley, 1971).

La Palinología puede ayudar en las interpretaciones paleoclimáticas, dado que utiliza características especiales de las plantas como son la alta producción 
de polen y esporas, su amplia dispersión en el registro fósil, su gran resistencia a la destrucción y la permanencia de características morfológicas en los gramos de polen; sin embargo, se debe dar una gran importancia a la sistemática en la determinación del polen y las esporas fósiles sobre la base de sus similitudes con las modernas, por esta razón un herbario debe contar con los servicios de una Palinoteca.

También se debe prestar atención a la litología de los sedimentos o unidades de roca en donde se colectan las plantas y especialmente donde se obtienen el polen y las esporas, como una fuente de datos para inferir el paleoclima.

Sin embargo, es necesario tomar en cuenta, en los estudios palinológicos, que el polen colectado en una determinada localidad no representa fielmente la comunidad adyacente de plantas, ni siquiera cualitativamente. Esta conclusión fue obtenida de estudios hechos en lagos para determinar la relación del polen depositado en los sedimentos con la vegetación que los rodea (Davis, 1963, 1965; in Dorf 1970). Tal falta de representación se debe principalmente a cuatro factores:

1.-Contaminación debida al transporte de polen de regiones remotas por aire o por agua.

2.-Abundante representación de ciertas formas polínicas debida a una sobreproducción de polen de algunas plantas tales como Pinus y Corylus.

3.-Subrepresentación de algunas formas, debido a que son poco productoras de polen, tales como Acer o bien formas que producen polen que es poco resistente a los procesos selectivos de fosilización como Tilia.

4.- - Contaminación debida al aporte de granos derivados de otros sedimentos por erosión acarreados y redepositados por el agua en un lago.

Le Clercq (1956) hace notar que los períodos de profundos cambios en las floras fósiles están relacionados con grandes acontecimientos geológicos: movimientos orogénicos, grandes transgresiones marinas, glaciaciones, cambios importantes del equilibrio químico, etc. Antes, durante o después de estos acontecimientos aparecen nuevas formas (tipogénesis) y desaparecen otras, hechos que están en íntima relación con la evolución y adaptación de las plantas. Datos micro y macropaleobotánicos confirman lo anterior y son índices de la variación y adaptación de la flora actual. Muchos grupos de plantas vienen desde hace miles de millones de años hasta el presente, con poca o mucha variación y otros han desaparecido de la faz de la tierra tiempo ha. El estudio de la flora actual nos permite comparar los grados de cvolución de ella y la Estratigrafía 
nos ayuda a situarla en el tiempo y en el espacio con precisión, permitiendo saber sus relaciones con los demás grupos actuales conocidos. Los herbarios son, por tal motivo, una fuente muy grande de información para conocer la historia, diversificación y distribución de los vegetales.

Dorf (1970) señala que desde hace poco se ha visto con claridad que ciertos caracteres morfológicos de las hojas de las Dicotiledoneas leñosas son indicadores del clima, independientemente del taxon al cual las hojas pertenecen. Ya desde principios de siglo (cita Dorf) Bailey y Sinnott encontraron que existe una clara correlación entre los caracteres fácilmente observables de las hojas y las condiciones del clima en el cual las plantas crecen. Estos investigadores fueron los primeros en reconocer la importancia de estas observaciones en el estudio del paleoclima basado en caracteres de hojas fósiles independientemente de sus relaciones taxonómicas. Sin embargo, Dorf considera que dentro de los caracteres morfológicos de las hojas en las interpretaciones paleoclimáticas existen unos más importantes que otros. Las tres características, en orden de importancia, son: 1) características marginales, 2) tamaño, 3) nerviación.

1.-En general los bordes completos son característicos de hojas del $75 \%$ de las especies de Dicotiledóneas leñosas de bosques húmedos de las tierras bajas tropicales. Sin embargo, Dorf hace notar que en algunos hábitos de alta montaña, y árticos muy fríos, en áreas secas como desiertos y planicies salinas, dominan las dicotiledóneas con limbos completos. Un conjunto fósil de este tipo podrá reconocerse debido al pequeño tamaño de las hojas. Por otro lado, las hojas no enteras aumentan del $20 \%$ en las tierras bajas tropicales al $80 \%$ en los bosques de tierras templadas frías húmedas de altas latitudes y de altas montañas.

2.- Los megafilos dominan en las tierras bajas tropicales en regiones con precipitación pluvial adecuada durante todo el año. Por ejemplo, en Panamá el $56 \%$ de las Dicotiledóneas leñosas tienen megafilos mientras que en California únicamente el $27 \%$. Los microfilos dominan en bosques templados (60$90 \%$ ) aumentando este carácter hacia los lugares de mayor latitud y altitud.

3.--Según Dorf, en las Dicotiledóneas leñosas, la nervadura es normalmente pinnada o palmeada. La nervadura pinnada es más común en bosques tropicales (aproximadamente 85\%) que en los templados (aproximadamente $75 \%$ ). Sin embargo, la combinación de nerviación palmeada con lóbulos palmeados es muy rara en los trópicos, (menos de 2\%, como en Acer y Platanzs), pero bastante común en los medios ambientales templados, (5-10\%). La nerviación palmeada en hojas de forma más o menos acorazanada, es típica de 
lianas trepadoras, comunes de climas húmedos tropicales y muy rara (menos de 2\%) en bosques templados (según Wolfe, 1969; in Dorf, 1970). La densidad de la nerviación y la nerviación terciaria, también tienen gran relación con diferencias climáticas. Según Wolfe, las hojas de Dicotiledóneas leñosas tropicales, tienen más nervios que las de tierras templadas. Desgraciadamente en las hojas fósiles es muy difícil determinar estos últimos caracteres.

Como hemos visto, los caracteres de las plantas indicadoras de climas, están representados en los fósiles únicamente de manera morfológica. Por lo anterior es necesario hacer una buena comparación del material fósil con el material actual de los herbarios, para poder hacer una identificación exacta de los taxa presentes al estado fósil. En la determinación de los climas del Cretácico Medio y del Terciario, se emplean generalmente hojas fósiles de Dicotiledóneas y su precisa identificación depende, según Dorf, de una cuidadosa consideración de los caracteres antes citados y del estudio microscópico de detalles de la cutícula de las hojas fósiles incluyendo el tamaño y el o los aspectos de los estomas cuando éstos están conservados. La comparación de semillas, madera, flores y frutos será de gran utilidad para aumentar el grado de precisión en la identificación.

El estudio de las polenesporas extraídas de los sedimentos que contienen los macrofósiles es de gran importancia, ya que, en primer lugar, corrobora las c'eterminaciones hechas de los megafósiles y en segundo lugar aporta nuevos datos de plantas que no pudieron ser fosilizadas, agrandando de esta manera la cantidad de datos taxonómicos que deberán ponerse en juego para determi. nar el paleoclima. Si lo anterior fuera poco es posible determinar, por el método palinológico, especies alóctonas indicadoras de la topografía y clima circundante, tipos de sedimentación, medios ambientes de depósito, lejanía de la línea de costa, edad de los sedimentos, grado de metamorfismo de la roca, etc., datos de gran valor geológico. Los granos de polen neógenos y cuaternarios pueden ser relacionados con plantas actuales a nivel de especie, los cretácicos y paleógenos a nivel de género o de familia, los pre-cretácicos a nivel de familia o taxa más grandes, aunque algunos pertenecen a taxa exclusivamente fósiles y por lo tanto, no pueden ser tomados en consideración en la interpretación de paleoclimas. Sin embargo, algunos de estos taxa están asociados con sedimentos que presentan otros datos indicadores del clima pasado. En este caso se encuentran algunas especies y géneros de polen de Coníferas, de Cycadales, de Bennettitales y de Angiospermas fósiles. Otra de las ventajas del estudio paleopalinológico es la siguiente: permite hacer un análisis tan preciso como se quiera de grandes secciones de sedimentos tanto continentales como marinos, 
en las cuales se ven los cambios litológicos relacionados con los cambios botánicos cuantitativos y cualitativos. Esto permite determinar cambios climáticos sucesivos durante un lapso bastante grande, que puede incluir una época, un período o una era. Estos cambios climáticos pueden ser corroborados por estudios de otros microfósiles vegetales tales como fitoclastos, Dinoflagelados y sus quistes, Acritarcas, Diatomeas, Carófitas, etc., cuya abundancia y tipos muchas veces depende de las condiciones climáticas reinantes en el continente y en el océano representadas en forma de cambios de salinidad, de solubilidad de algunas sales, etc.

Al igual que los caracteres morfológicos de las hojas, las polenesporas presentan rasgos morfológicos indicadores de las condiciones ecológicas del lugar en donde viven las plantas que las producen.

La mayoría de los caracteres evolutivos de las polenesporas han sido determinados en base a estudios de materiales actuales provenientes de grandes herbarios. Este tipo de estudios fundaron la palinología, ya que desde mediados del siglo xvi se hacen descripciones morfológicas del polen. Investigadores famosos y laboratorios en diferentes partes del mundo se dedican al estudio de polenesporas con el fin de encontrar en ellas las bases de la evolución de las plantas, las bases de una nueva clasificación de las mismas o de un nuevo acomodo de los vegetales de acuerdo a su tipo de polen. Todos estos puntos relacionados con el estudio de las polenesporas fósiles nos permiten determinar los niveles de aparición de los diferentes caracteres morfológicos en el tiempo geológico y la antigua distribución de las plantas.

Es claro que desde el punto de vista de la determinación de factores climáticos y ecológicos por medio de la interpretación de los caracteres morfológicos de las polenesporas, debamos hacer una diferenciación jerarquizada de ellos. Los más importantes son:

1.-El espesor de la pared: ésta es más gruesa en plantas de regiones áridas. Las plantas acuáticas presentan por lo general paredes delgadas y celulósicas.

2.-De adaptación al medio de transporte acuoso o aéreo. En algunas esporas se presenta una separación de las dos capas principales de la pared que se llenan de aire; esta cámara les permite desplazarse sobre el agua. En el polen de Coníferas, la separación de las membranas furma vesículas o sacos que se llenan de aire y que les permiten ser transportados con facilidad por el viento.

3.-De la escultura y ornamentación. Los tipos de ornamentación están intimamente ligados al tipo de agentes vectores que efectúan la distribución de las polenesporas. El polen de las plantas anemófilas es liso, oponiendo así me- 
nor resistencia al aire. El de plantas zoófilas presenta espinas, huecos, bastones, pelos, etc. que permiten que el polen se adhiera fácilmente al animal vector. El de plantas hidrófilas presenta retículos en donde se adhieren burbujas que les permiten flotar fácilmente sobre el agua.

4.- El tamaño de las polenesporas está estrechamente relacionado con el tipo de agente vector. Las esporas son generalmente grandes porque utilizan la gravedad o el agua para su distribución. El prepolen de las Pteridospermas era grande, pues empleaba la fuerza de gravedad. El polen de Gimnospermas es menos grande que el anterior, pues utiliza el viento como agente vector; el de las Angiopermas anemófilas es el más pequeño de todos; el de las zoófilas es en general muy grande pues emplea agentes vectores potentes (insectos, aves, mamíferos); el de plantas acuáticas es también grande y en algunos casos alcanza dimensiones gigantescas $(2500 \mu$ en el caso del género Zostera).

Como vemos, el estudio de las polenesporas actuales, tomando en consideración que se conocen todos los datos de la planta madre, es de capital importancia para basar los estudios de las polenesporas fósiles. Como hemos citado, los caracteres morfológicos de ellas, que en su mayoría pueden ser determinados, deben ser relacionados con los datos ecológicos para llegar a conclusiones del mismo tipo en el pasado.

\section{CONCLUSIONES}

Como los fósiles vegetales deben ser comparados con plantas actuales conservadas en forma adecuada, el acervo de estas plantas debe ser muy completo y en el mejor estado de conservación que permita su estudio morfológico, anatómico, etc. Además, de cada ejemplar deben conocerse: su clasificación, su grado de evolución y las condiciones ecológicas que permitan inferir su grado de adaptación. Todos estos datos del presente favorecen la formulación de hipótesis al paleobotánico, respecto al grado de evolución y a las condiciones ecológicas existentes en el pasado, cuando se encuentre semejanza entre un fósil vegetal con una planta actual. Esta semejanza puede ser no únicamnte a nivel macroscópico sino también a nivel microscópico. Por eso es necesario que en un herbario existan ejemplares con las partes vegetativas y reproductivas de una planta.

Un herbario deberá comprender dos secciones básicas, una correspondiente a plantas macroscópicas y otra microscópicas. Ya citamos anteriormente la importancia del estudio de las plantas microscópicas y de las partes microscó- 
pics de las plantas macroscópicas, por lo que es de gran importancia para los estudios paleobotánicos contar con el acceso a herbarios lo más completos posible y hacer comparaciones de macro y microfósiles con las plantas ahí almacenadas. La colección de preparaciones de polen y esporas de las plantas de un herbario toma el nombre de Palinoteca. Actualmente, reviste una gran importancia el estudio del polen y las esporas fósiles y puede decirse con toda propiedad que la gran mayoría de los estudios paleobotánicos actuales pertenecen a estudios de polenesporas fósiles, fitoclastos y microorganismos vegetales marinos fósiles. Esto se debe al gran desarrollo de las aplicaciones de la Palinología dentro de la Geología en general y en particular en la prospección de energéticos. Pocas ciencias han tenido un desarrollo tan espectacular y pocas han estado tan ligadas a aplicaciones tan amplias dentro de diferentes campos del dominio técnico-científico.

Según Traverse (1955) en su trabajo del Lignito Brandon de Vermont cualquier persona que estudie las plantas del Terciario y del Cuaternario debe tener una colección de referencias. La fineza en la identificación hecha de plantas terciarias depende sobre manera de la calidad de la colección de comparación de las especies vivientes, de cómo y por quién han sido clasificadas y la de la sistematización de la información así obtenida.

De todos los órganos de plantas del Terciario posiblemente aquéllos que permiten una clara identificación son los granos de polen y esporas ya que poseen características morfológicas que permiten hacer una diagnósis correcta. El número de aberturas y su estructura, el número de capas, la escultura externa y aún la interna así como la forma y el tamaño, son datos que permiten clasificar los granos de polen y compararlos con los que se tengan en un herbario del área en cuestión.

Ahora bien, la colección de referencia puede constar de laminillas de material microscópico listas para observación y comparación o simplemente consistir de frasquitos con el material en ácido acético, listo para efectuar en cualquier momento la confección de las laminillas después de realizar la técnica de la acetólisis; esto dependerá de la preferencia de cada encargado del herbario o del espacio disponible y personal con que se cuente para realizar estas tareas, ya que el enorme número de géneros del Terciario ameritaría una colección por demás completa para que realmente diera la utilidad deseada.

Si se opta por hacer preparaciones microscópicas, éstas deben ser hechas usando la técnica más adecuada y permanente, seleccionando del material del herbario las yemas de las flores que de preferencia hayan sido colectadas justo antes de la antesis, ya que el polen estará casi maduro y en cantidades nece- 
sarias. Cada preparación llevará el número del ejemplar del cual fue extraido el polen a fin de relacionar ambos, independientemente de que se elija otra forma de catalogarlas. Esta técnica ya es empleada en diferentes instituciones de investigación botánica.

La importancia que tiene una palinoteca como parte de un herbario se basa en que:

a) El polen y las esporas son depositarios genéticos de la futura planta.

b) Son útiles para fines de comparación con polen y esporas fósiles permitiendo diferenciar, separar, identificar géneros y especies o taxa mayores en base principalmente a stu morfología utilizando como medio accesorio atlas fotográficos.

\section{BIBLIOGRAFIA CONSULTADA}

Alvin, K. L. 1966. Two cristate megaspores from the Lower Carboniferous of Scotland. Palaeontology $9(3): 488-491$.

Andrews, H. H., Jr. 1961. Studies in Paleobotany. John Wiley \& Sons. Inc. pp. 487.

Archangelsky, S. 1970. Fundamentos de Paleobotánica. Serie Técnica y Didáctica No. 10. Universidad Nacional de La Plata. pp. 347.

Benson, L. 1957. Plant Classification. D. C. Heath and Co. Boston. pp. 688.

Boulter, M. C. 1971. A palynological study of two of the Neogene plants beds in Derbyshire. Bull. Brit. Mus. (Natur. Hist.) Geol. 19(7):361-410.

Chaloner, G. W. 1967. Spores and land-plant evolution. Rev. Palaeobotan. Palynol. 1(1/4): 83.94.

Delevoryas, T. 1966. Diversificación vegetal. Cía. Editorial Continental, S. A. 2a. reimpresión 1968. pp. 193. , 1962. Morphology and Evolution of Fossil Plants. Holt, Reinhart and Winston,
Inc. pp. 189.

Dorf, E. 1964. The use of fossil plants in Paleoclimatic Interpretations. In: Problems in Climatology: 13-30. Edit. Nairn. Interscience Publishers.

1970. Paleobotanical evidence of Mesozoic and Cenozoic climatic changes. Proceedings of the North American paleontological convention. Part. D. Paleoclimatology. Allen Press Inc. Lawrence, Kansas: 323:346.

Erdtuan, G. 1963. Palynology. In: Advances in Botanical Research: 149-208. Edit. Academic Press. Inc.

- 1969. Handbook of Palynology. Hafner Publishers Co. pp. 486.

Font Quer, P. 1953. Diccionario de Botánica. 2a. reimpresión 1965. Editorial Labor, S. A. Barcelona. pp. 1244.

Fosberg, F. R. and SAchet, M. E. 1965. Manual for Tropical Herbaria. Regnum Vegetabile 39:1-132.

Herm, R. \& Perreau, J. 1959. Avant-propos. Pollen et Spores I(1) :5-6.

-, 1971. Etude ornamentale de basidiospores au microscope electronique a balayage. In: Scanning Electron Microscopy: 251-284. Edit. Heywood, V. H. Academic Press.

Kay, M. \& Colbert, E. H. 1965. Stratigraphy and Life History. John Wiley \& Sons. pp. 736.

Krumbern, W. C. \& Stoss, L. L. 1963. Stratigraphy and Sedimentation. W. H. Freeman \& Co. pp. 660. 
Le Clerce, S. 1956. Evidence of vascular plants in the Cambrian. Evolution 10:109-114.

Lemman, H. 1972. Classification and explanation in Biology. Taxon 20(2/3) :257-268.

Moore, C. R. Lalicker, G. C. \& Fischer, A. G. 1952. Invertebrate Fossils. McGraw Hill Book Co. Inc. pp. 766.

Moret, L. 1943. Manuel de Paléontologie Végétale. Masson et Cie. pp. 230.

Morton, V. \& Stern, L. W. 1966. The United States National Herbarium. Plant Science Bulletin 12(2):1-4.

Narr, P. K. K. 1970. Pollen morphology of Angiosperms. A Historical and Phylogenetic Study. Scholar Publishing House National Botanic Gardens, Lucknow, India. pp. 160.

Noailles, C. M. 1969. La evolución botánica. Ed. Martínez Roca, S. A. Barcelona. pp. 188.

Reyre, Y. 1971. Interprétation Botanique des Pollens Inaperturés du Mésozoique Saharien. Essai de Classification d'après l'Observation en Microscopie Electronique à Balayage. In: Scanning Electron Microscopy: 145-154. Edit. Heywood, V. H. Academic Press.

Samylina, V. A. 1968. Early Cretaceous angiosperms of the Soviet Union based on leaf and fruit remains. J. Linn. Soc. (Bot.) LXI (384) :207-218.

Solbrig, T. O. 1966. Evolution and systematics. The Macmillan Co. New York. pp. 122.

Stuckey, L. R. 1971. The first public auction of an American Herbarium including an account of the fate of the Balwin, Collins, and Rafinesque Herbaria. Taxon 20(4): 443-459.

Sylvester-Bradley, P. C. 1971. The reaction of systematics to the revolution in Micropaleontology. In: Scanning Electron Microscopy: 95-112. Edit. Heywood, V. H. Academic Press.

Takhtajan, A. 1959. Flowering plants. Origin and Dispersal. Smithsonian Institution Press. pp. 310.

Traverse, A. 1955. Pollen Analysis of the Brandon Lignite of Vermont. Bureau of Mines. Report of Investigation 5151. U. S. Department of the Interior. pp. 107.

Tschudy, R. H. \& Scott, R. A. 1969. Aspects of Palynology. Wiley-Interscience. pp. 510.

Théobald, N. \& Gama, A. 1959. Stratigraphie. G. Doin et Cie. pp. 385.

Thompson, P. A. 1972.The role of the botanic garden. Taxon 21(1):115-119.

Wesley, A. 1963. The Status of Some Fossils Plants. In: Advances in Botanical Reséarch: 1-68. Edit. Preston, R. D. Academic Press. 